\title{
Effects of Nonischemic Hypoxia on Jejunal Mucosal Structure and Function: Study of an Experimental Model in Dogs
}

\author{
MOSHE BERANT, URI ALON, DITZA ANTEBI, ERIC DIAMOND, HEDWIG KOERNER, AND \\ DANIEL MORDECHOVITZ \\ Department of Pediatrics [M. B., U. A., D. A.], Laboratory of Clinical Biochemistry [E. D.], Institute of \\ Pathology [H. K.], and Experimental Surgery Unit [D. M.], Rambam Medical Center and Faculty of Medicine, \\ Technion-Israel Institute of Technology, Haifa, Israel
}

\begin{abstract}
A model of nonischemic hypoxia of the jejunum was designed in dogs, by shunting of blood from the inferior vena cava directly into the regional mesenteric arterial supply, thereby lowering the $\mathrm{PaO}_{2}$ of the blood that reached the jejunal wall from $98.6 \pm 3$ to $62 \pm 5 \mathrm{~mm}$ Hg. Absorption rates of sodium, glucose, fructose, glycine, and the dibasic aminoacid lysine were studied by in situ luminal perfusion of a $30-\mathrm{cm}$ proximal jejunal segment with a bicarbonate buffer solution containing phenol red as a nonabsorbable marker for determination of water fluxes. During periods of control, hypoxia, and after discontinuation of the venoarterial admixture (recovery), effluent perfusate was collected and mucosal biopsies were obtained for assay of lactase, maltase and sucrase activity, mucosal ATPase activity and ATP content, and for light- and electron microscopic examination. Mesenteric supply with hypoxic blood was associated with a significant inhibition of $\mathrm{Na}^{+}, \mathrm{K}^{+}$-ATPase activity $(p<0.001)$ and a rise in mucosal ATP content $(p<0.05)$. There was a significant reduction in the absorption rates of sodium $(p<0.001)$, glucose, and glycine $(p<0.01)$, but no change in the transport of fructose and of lysine. Brush border enzymes were unaltered. The histological appearance of the mucosa remained normal throughout the experiment, but on electron microscopy a distinct swelling of the enterocyte mitochondria was noted during the hypoxia period. Discontinuation of the venoarterial shunt and return to well-oxygenated mesenteric blood supply to the small gut was associated with a recuperation of $\mathrm{Na}^{+}, \mathrm{K}^{+}$-ATPase activity, decrease of $\mathrm{mu}$ cosal ATP content, improvement of mucosal absorption capacity, and disappearance of the mitochondrial swelling. It is concluded that nonischemic hypoxia of the jejunum can impair $\mathrm{Na}^{+},-\mathrm{K}^{+}$-ATPase-related jejunal absorption functions in the absence of apparent structural damage. (Pediatr Res 20: 1143-1146, 1986)
\end{abstract}

The small intestinal mucosa is very sensitive to the adverse effects of oxygen deprivation. Diverse experimental investigations have demonstrated a variety of alterations-microcirculatory, morphological, and functional-that may be provoked by small intestinal hypoxia (1-7). Most of the studied conditions, however, involve an impairment of the blood supply to the gut,

Received April 4, 1986; accepted June 17, 1986.

Correspondence to Prof. Moshe Berant. Department of Pediatrics, Rambam Medical Center, Haifa 35254, Israel.

' Presented in part at the Meeting of the Society for Pediatric Research, May 59, 1986, Washington, D.C. such as arterial occlusion $(1,2)$ or hypotension (3-5), or circumstances associated with cardiocirculatory fluctuations $(6,7)$; these situations entail a definite element of ischemia, which can by itself set off a train of injurious events (8).

We studied the effects of nonischemic hypoxia. In our experimental model in dogs, we lowered the oxygen content of the arterial blood supplied directly to the proximal jejunum by introducing, in situ, an admixture of venous blood. We could thus observe the influence of mild to moderate nonischemic hypoxia on jejunal mucosal morphology and function.

\section{MATERIALS AND METHODS}

Adult mongrel dogs weighing $15-20 \mathrm{~kg}$ were anesthetized with Nembutal given intravenously in a dose of $20 \mathrm{mg} / \mathrm{kg}$. During the experiment the dogs had an endotracheal tube in place connected to a mechanical respiration pump (Model Harvard Apparatus, S. Natick, MA). Ringer's lactate solution was given intravenously at a rate of $10 \mathrm{ml} / \mathrm{kg} / \mathrm{h}$, and the dogs' respiratory and cardiocirculatory status was constantly monitored. Jejunal mucosal function was assessed by luminal perfusion in situ of a proximal jejunal segment and calculation of the transport rates of sodium, monosaccharides, and aminoacids, and by assay of brush border enzymes, mucosal ATPase activity, and mucosal ATP content. Concurrently with perfusion, regional hypoxia was induced as the mesenteric arterial vessel supplying the perfused jejunal loop received an admixture of venous blood from the dog's inferior vena cava.

By a midline abdominal incision, the proximal jejunum was exposed and a $30-\mathrm{cm}$ segment was prepared for luminal perfusion. The mesenteric arterial branch supplying this segment and the neighboring intestinal loops was identified; a 20-gauge QuickCath cannula was inserted without ligation and secured by stitching to the perivascular tissue, thus fully preserving arterial flow. Injection of dilute aqueous methylene blue solution caused a fleeting bluish discoloration of the proximal gut, which allowed us to assess the length of intestine supplied by the cannulated artery and to ascertain that the jejunal loop to be perfused was fully included. A distal arterial branch next to the intestinal wall and just distal to the perfused loop was cannulated with a 25 gauge Quick-Cath tube with a heparin lock, for serial blood sampling and determination of local blood gases. An adjacent branch was similarly cannulated and connected to a U-type manometer for monitoring the local blood pressure. An indwelling catheter was inserted into the inferior vena cava. The experimental set-up is shown in Figure 1.

The lumen of the jejunal loop was perfused with KrebsHenseleit bicarbonate buffer, propelled by a Harvard peristaltic pump (Millis, MA) at an average rate of $6 \mathrm{ml} / \mathrm{min}$. The perfusion 
solution included $123 \mathrm{mmol} /$ liter sodium, $4 \mathrm{mmol} /$ liter potassium, $45 \mathrm{mmol} /$ liter of either glucose or fructose, and $10 \mathrm{mmol} /$ liter of either glycine or the dibasic aminoacid lysine, labeled with $20 \mu \mathrm{Ci}$ /liter [ ${ }^{3} \mathrm{H}$ ]-amino acid (New England Nuclear, Boston, MA). Phenol red $20 \mathrm{mg} /$ liter was added as a nonabsorbable marker for estimation of water fluxes (9). The solution was continuously bubbled with a mixture of $95 \% \mathrm{O}_{2}$ and $5 \% \mathrm{CO}_{2}$, yielding a $\mathrm{PCO}_{2}$ of about $40 \mathrm{~mm} \mathrm{Hg}$; the $\mathrm{pH}$ was kept at 7.4 and the osmolarity at $300 \mathrm{mosmol} / \mathrm{liter}$. Each sugar and aminoacid was tested in groups of six dogs.

Jejunal perfusion was performed under three conditions: 1) control period, 2) hypoxia period, and 3) recovery period.

Control period. After allowing $2 \mathrm{~h}$ for recovery from the initial surgical procedure, effluent fluid from the jejunal perfusion was collected over $1 \mathrm{~h}$ in 20-min aliquots, while the intestine received its normal arterial blood supply. The mean blood flow in the main supplying vessel was $278 \pm 11 \mathrm{ml} / \mathrm{min}$. The arterial blood next to the intestinal wall had a pH of $7.36 \pm .03, \mathrm{PaO}_{2} 98.6 \pm$ $3 \mathrm{~mm} \mathrm{Hg}$, and bicarbonate $23 \pm 2 \mathrm{mmol} /$ liter.

Hypoxia period. The catheter in the inferior vena cava was connected with the tubing of the cannula in the mesenteric artery, and venous blood was shunted from the vein into the artery, drawn and propelled by a Holter peristaltic pump at an average rate of $80 \mathrm{ml} / \mathrm{min}$. The $\mathrm{PaO}_{2}$ in the blood that reached the intestinal wall decreased to $62 \pm 5 \mathrm{~mm} \mathrm{Hg}$; the $\mathrm{pH}$ and bicarbonate values were unchanged. Immediately after activating the venoarterial shunt, the locally measured blood pressure rose temporarily from $125 \pm 6$ to $134 \pm 7 \mathrm{~mm} \mathrm{Hg}$, with a decrease in blood flow to $265 \pm 15 \mathrm{ml} / \mathrm{min}$. After $5 \mathrm{~min}$, all measurements had reverted to their previous control values and remained stable throughout.

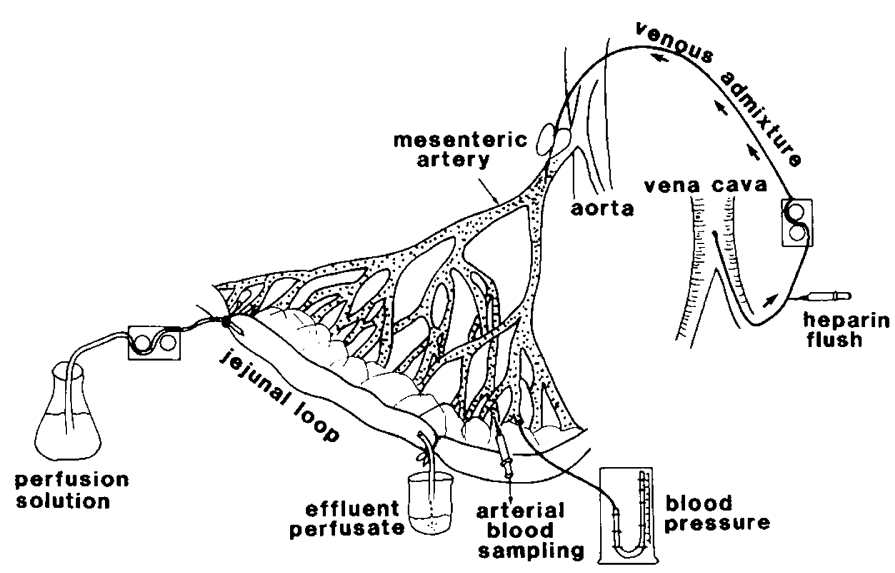

Fig. 1. Diagram of the experimental model. Luminal perfusion of a proximal jejunal segment and shunting of blood from the inferior vena cava into the mesenteric arterial supply to the perfused jejunal loop is shown.
After $1 \mathrm{~h}$ of equilibration, 20-min aliquots of effluent perfusate were collected over $1 \mathrm{~h}$.

Recovery period. The caval-mesenteric shunting was discontinued and $1 \mathrm{~h}$ of equilibration was allowed, while the jejunum again received its normal arterial blood supply $\left(\mathrm{PaO}_{2} 97 \pm 4 \mathrm{~mm}\right.$ $\mathrm{Hg}$ ). Effluent perfusate was subsequently collected over $3 \mathrm{~h}$ in 20 -min aliquots. During the last hour of this period, the mean $\mathrm{pH}$ of the arterial blood next to the intestinal wall fell to $7.25 \pm$ 02 and remained at this level for the rest of the study. In parallel, blood from the femoral artery had a $\mathrm{pH}$ of $7.27 \pm 0.03, \mathrm{PaO}_{2} 96$ $\pm 4 \mathrm{~mm} \mathrm{Hg}$, and bicarbonate $18 \pm 3 \mathrm{mmol} /$ liter.

Blood gases were measured with a blood gas analyzer (Corning model 168, Halstead, Essex, UK); arterial blood flow was determined with an electromagnetic flowmeter (Carolina Electronics Corp., King, NC).

The jejunal transport rates of sodium, glucose, fructose, glycine, and lysine were calculated from the difference of their concentrations in the perfusion solution and in the effluent, and corrected for water flux, length of perfused segment, and pumping rate. The concentration of sodium was measured by an ionspecific electrode (Beckman Instruments ASTRA analyzer, High Wycombe, Bucks, UK); glucose, by a glucose oxidase method (Beckman Instruments ASTRA analyzer); fructose, by the method of Davis and Gander (10); glycine and lysine were determined by $\left[{ }^{3} \mathrm{H}\right]$ liquid scintillation counting (Beckman LS230); and phenol red was measured by the method of Miller and Schedl (9). The amount of phenol red recovered in all samples of effluent did not differ statistically from $100 \%$.

Toward the termination of each stage of the experiment, biopsy samples from the wall of the jejunum just distal to the perfused loop and supplied by the same mesenteric vessel were obtained. From these cut-out biopsy patches, a portion was processed by standard procedures for study by light and electron microscopy (Zeiss EM9A, Oberkochen, W. Germany). From the remaining piece, the mucosa was carefully scraped off with a glass slide, immediately frozen, and stored at $-60^{\circ} \mathrm{C}$ until assayed for mucosal enzyme activity and mucosal ATP content, 5 to 7 days later. The brush border enzymes lactase, sucrase, and maltase were measured by the method of Dahlqvist (11). Mucosal ATPase activity was assayed by the method of Kramer et al. (12); ATP content was determined by the method of Lowry and Passonneau (13). The protein content of the mucosa was measured by the method of Bradford (14).

The data obtained were tested for statistical significance by analysis of variance for repeated measures.

\section{RESULTS}

Jejunal transport (Fig. 2). During the 2 nd h of hypoxia water absorption fell from (mean \pm SD) $11.4 \pm 3.8$ to $2.2 \pm 4.7 \mu \mathrm{l} /$ $\mathrm{min} / \mathrm{cm}(p<0.01)$. The mean absorption rates of sodium, glucose, and glycine were significantly decreased $(p<0.001$ versus control). Over the period of recovery a gradual improvement in transport was noted, and during the 3rd $\mathrm{h}$ of collection of effluent perfusate ( $4 \mathrm{th} \mathrm{h}$ after resumption of supply with well
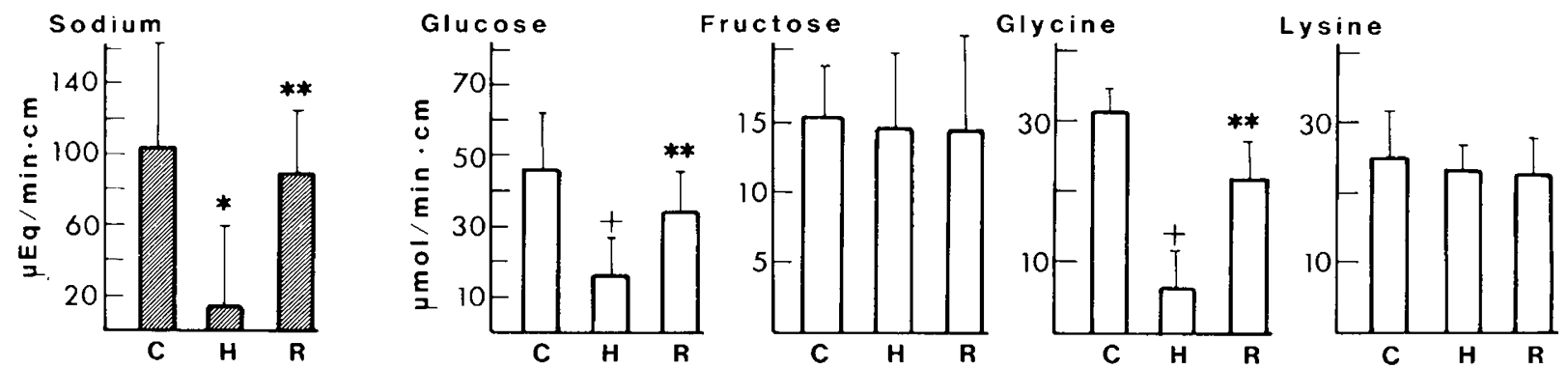

Fig. 2. Jejunal mucosal transport rates (mean $\pm \mathrm{SD}$ ) of sodium, glucose, fructose, glycine and lysine, during the control $(C)$, hypoxia $(H)$, and recovery $(R)$ periods. Sodium, glucose and glycine transport is significantly reduced by hypoxia; improvement occurs during recovery period. Fructose and lysine absorption is not altered. ${ }^{*} p<0.001$ versus $\mathrm{C} ;{ }^{+} p<0.01$ versus $\mathrm{C} ;{ }^{* *} p<0.01$ versus $\mathrm{H}$. 
oxygenated arterial blood) water absorption had increased to (mean $\pm \mathrm{SD}) 7.8 \pm 3.6 \mu \mathrm{l} / \mathrm{min} / \mathrm{cm}(p<0.02$ versus hypoxia), and the mean absorption rates of sodium, glucose, and glycine had risen significantly ( $p<0.01$ versus hypoxia). The transport of fructose and of lysine was unaffected by hypoxia, and their mean transport rates did not change significantly over the three experimental periods.

Jejunal mucosal enzymes. Hypoxia did not affect the specific activity of the studied brush border enzymes lactase, maltase, and sucrase; their respective values were similar during the three experimental periods. In contrast, the activity of the jejunal mucosal $\mathrm{Na}^{+}, \mathrm{K}^{+}$-ATPase was significantly depressed $(p<0.001$ versus control) during the $2 \mathrm{nd} h$ of hypoxia; total ATPases remained unchanged and $\mathrm{Mg}^{++}$-ATPase was increased, but not significantly. At the 4 th $\mathrm{h}$ of the recovery period the activity of $\mathrm{Na}^{+}, \mathrm{K}^{+}$-ATPase rose significantly ( $p 0.05$ versus hypoxia), although it did not reach control values, and $\mathrm{Mg}^{++}$-ATPase decreased to nearly control values (Fig. $3 a$ ).
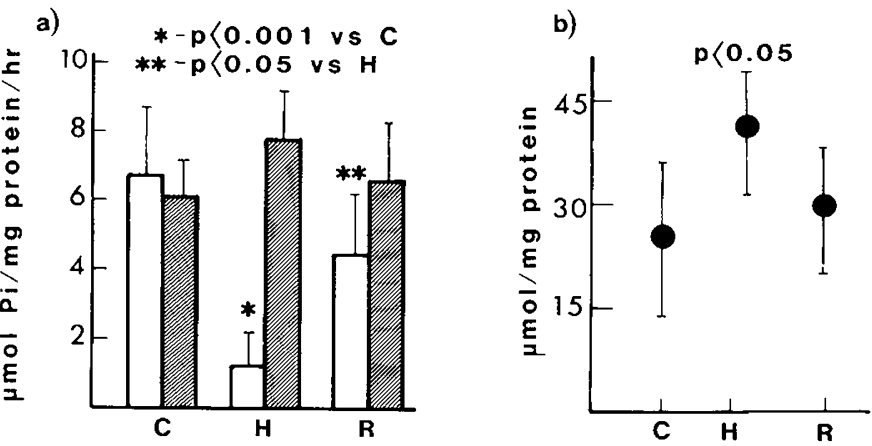

Fig. 3. $a$, mucosal ATPase activity (mean $\pm \mathrm{SD}$ ) during the control $(C)$, hypoxia $(H)$, and recovery $(R)$ periods. $\mathrm{Na}^{+}, \mathrm{K}^{+}$-ATPase activity $(\square)$ is significantly inhibited during hypoxia and rises during recovery. $\mathrm{Mg}^{2+}$ ATPase activity (逐) is increased during hypoxia. $b$, mucosal ATP content (mean $\pm \mathrm{SD}$ ) during the control $(C)$, hypoxia $(H)$, and recovery $(R)$ periods. ATP levels become significantly increased $(p<0.05)$ by hypoxia and return to near control levels during recovery.
Mucosal ATP content. This increased significantly $(p<0.05$ versus control) at the hypoxia period and decreased during the recovery period, mirroring the changes observed in the activity of $\mathrm{Na}^{+}, \mathrm{K}^{+}$-ATPase (Fig. $3 b$ ).

Mucosal histology and ultrastructure. The experimental procedure and the hypoxia induced by the venoarterial shunt did not cause any changes in the histology of the jejunal mucosa. The appearance of the epithelial layer, the villus/crypt proportions, the subepithelial structures, and the capillaries during the hypoxia and recovery periods were indistinguishable from those of the control period.

Electron microscopy demonstrated no alterations in capillaries and perivascular spaces, enterocyte arrangement, microvillar brush border, and intracellular organisation, at all three stages of the study. However, the period of hypoxia was associated with a distinct swelling of the enterocyte mitochondria (Fig. $4 b$ ), with a widening of the cristae and a decrease in matrix density. At the 4 th $h$ of recovery mitochondrial swelling was found to have completely subsided (Fig. 4c).

\section{DISCUSSION}

Hypoxia of the small gut has been reported to result in severe mucosal damage. There is denudation of villus epithelium, reduction in villus height and epithelial necrosis $(1-3)$, with edema and hemorrhagic infiltration of the lamina propria and marked vascular congestion (2). Microcirculation studies have demonstrated a complete arrest of the microperfusion of the villus core (2) and a significant increase in permeability of intestinal capillaries (3). A severe impairment of the transport capacity of the small intestinal mucosa has also been noted $(2,4,5)$, although mucosal ATPase were not affected (2).

The above mentioned observations have been derived from studies where hypoxia was induced by causing hypotension (35 ) or by different degrees of vascular occlusion (1-3), thus drastically reducing blood supply to the small intestine. In other works, hypoxia was provoked by submersion (6) or asphyxiation (7) of the experimental animal, and was also accompanied by a significant drop in intestinal regional blood flow. Because of the predominant element of ischemia involved, these investigational models may not be representative of the small intestinal effects
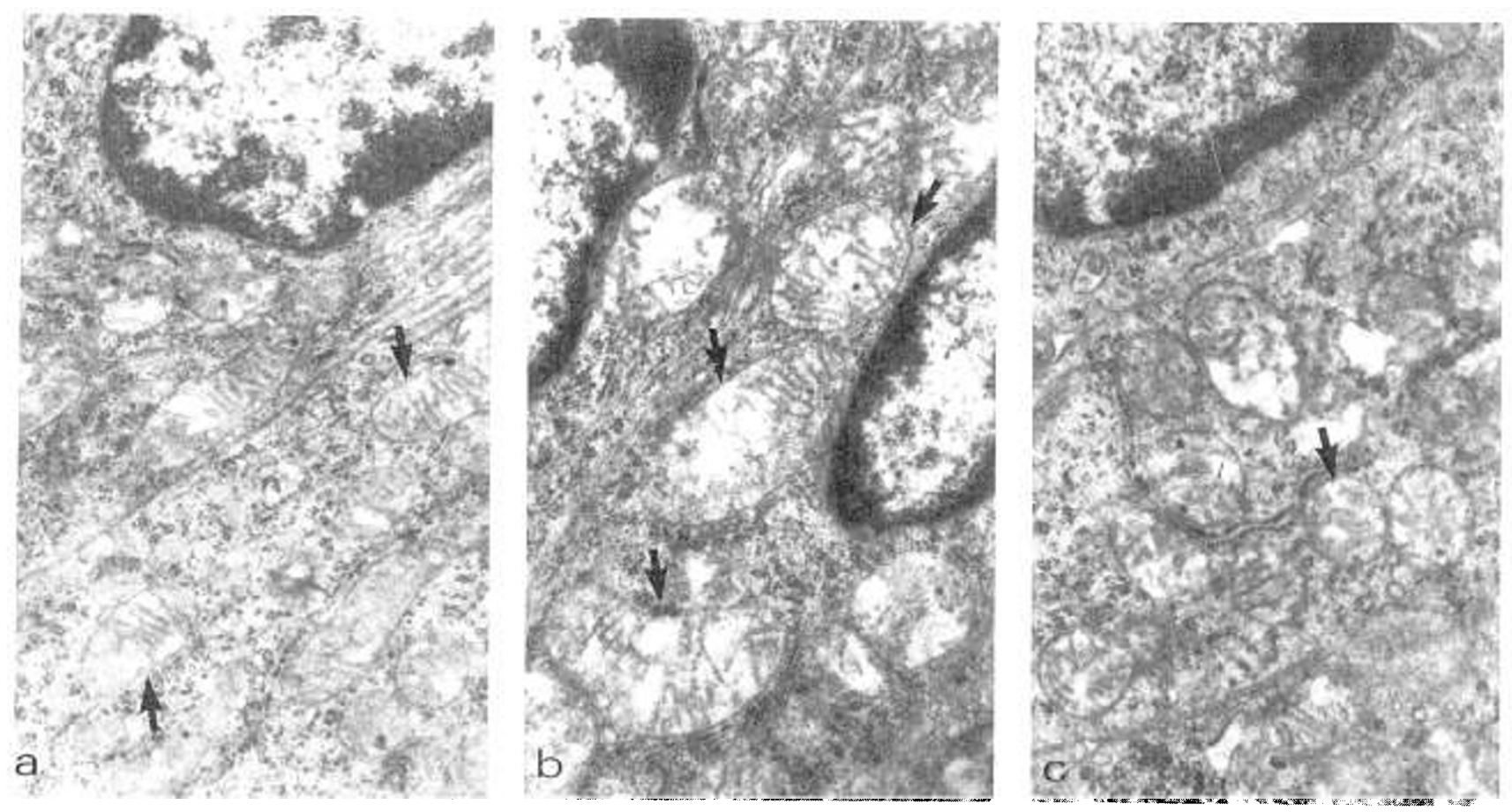

Fig. 4. Electron microscopic view of enterocyte mitochondria (arrows) (magnification, $\times 19,500$ ). a, appearance of mitochondria during the control period; $b$, mitochondrial swelling during the hypoxia period; $c$, regression of mitochondrial swelling during the recovery period. 
of hypoxia per se. An ischemic insult can give rise to a multiplicity of damaging events, notably the generation of oxygen-derived free radicals (8), and lead to morphological, microcirculatory, and functional derangements $(1,15)$. Perry et al. (16) designed a model of intestinal hypoxia in cats, where hypoxia $\left(\mathrm{PaO}_{2} \mathrm{~mm}\right.$ $\mathrm{Hg}$ ) was induced as the animals breathed $8 \% \mathrm{O}_{2}$ in $\mathrm{N}_{2}$, while blood flow to the studied loop of small gut was carefully maintained. These authors demonstrated an increase of intestinal capillary permeability during the hypoxia period.

In our experimental model for the study of nonischemic hypoxia, blood of a low $\mathrm{PO}_{2}$ was supplied directly to a proximal jejunal segment as its mesenteric arterial branch received an admixture of venous blood. Tissue hypoxia thus induced was of a mild to moderate degree, resembling that observed in actual clinical conditions, such as cyanotic heart disease or bronchopulmonary dysplasia. Blood flow to the gut was unhindered and the confounding effects of ischemia were thus avoided. Histological examination of the wall of the experimental jejunal loop showed no evidence of congestion, thrombosis, cellular swelling or tissue edema, indicating that the added blood volume was adequately accommodated. By inducing only regional hypoxia we could circumvent the respiratory, acid-base status and cardiocirculatory perturbations that acute systemic hypoxia might evoke.

In our study, the hypoxia period was associated with a significant depression of mucosal $\mathrm{Na}^{+}, \mathrm{K}^{+}$-ATPase activity and a rise in mucosal ATP content, concurrently with a significant reduction in the absorption rates of water, sodium, glucose, and glycine. The increase in mucosal ATP content during hypoxia probably reflects the inhibition of $\mathrm{Na}^{+}, \mathrm{K}^{+}$-ATPase activity and the associated decreased $\mathrm{Na}^{+}$transport (17), and suggests that the impairment of $\mathrm{Na}^{+}$transport is related to underutilization, rather than unavailability, of energy sources. It seems unlikely that increased capillary permeability during hypoxia (16) played a major role in the derangement of absorptive functions, as the transport rates of fructose and of lysine, which are not dependent on $\mathrm{Na}^{+}, \mathrm{K}^{+}$-ATPase activity, were unaffected by hypoxia. The activity of brush border enzymes remained unaltered, and $\mathrm{Mg}^{++}$. ATPase activity was not decreased. We found that the changes induced by the hypoxia period were reversible. Three hours after the venoarterial shunting was discontinued and the jejunal loop again received well-oxygenated arterial blood, there was a significant increase in mucosal $\mathrm{Na}^{+}, \mathrm{K}^{+}$-ATPase activity, along with a fall in mucosal ATP content and with a significant improvement of water, sodium, glucose, and glycine absorption-although not attaining the values of the control period. The failure of $\mathrm{Na}^{+}, \mathrm{K}^{+}$ATPase to regain its full activity may be ascribed to the metabolic acidosis that developed in the dogs during the last stage of the experiment, as the activity of this enzyme has been shown to be adversely affected by a low $\mathrm{pH}$ and low blood bicarbonate concentrations (18).

Our findings conform with those of Lifshitz et al. (18) who studied the effects of mild to moderate hypoxia by placing rats in an $\mathrm{O}_{2}$-poor environment. They too found a depression in jejunal mucosal $\mathrm{Na}^{+}, \mathrm{K}^{+}$-ATPase activity with a concomitant impairment in the absorption of sodium, glucose, and actively transported aminoacids, whereas fructose and lysine absorption was not affected.

In the present study, as in the work of Lifshitz et al. (18), the histological appearance of the jejunal mucosa during the period of hypoxia was indistinguishable from that of the control. We also did not note any changes during the recovery period. On the other hand, whereas Lifshitz et al. (18) could not find any ultrastructural alterations, electron microscopic examination of the epithelial cells in our material showed that the period of hypoxia was associated with a distinct swelling of the enterocyte mitochondria, a finding that might be related to the reduction in $\mathrm{Na}^{+}, \mathrm{K}^{+}$-ATPase activity and in $\mathrm{Na}^{+}$transport during the period of relative oxygen deprivation. The mitochondrial swelling observed during hypoxia was no longer seen after normally oxygenated blood supply to the jejunum was reestablished.

We conclude that a period of mild to moderate nonischemic hypoxia of the small intestine may depress mucosal $\mathrm{Na}^{+}, \mathrm{K}^{+}-$ ATPase activity and impair $\mathrm{Na}^{+}, \mathrm{K}^{+}$-ATPase-associated absorption in the absence of morphological mucosal alterations other than swelling of the enterocyte mitochondria. The observations derived from the present experimental model may offer some insights into the growth failure of children who have for example congenital cyanotic heart disease without cardiac decompensation, where impairment of intestinal absorptive capacity by the hypoxia could play a contributory role.

Acknowledgments. The authors thank Mr. Nahum Azaria for technical assistance and Ms. Ruth Rose for typing the manuscript.

\section{REFERENCES}

1. Parks DA, Bulkley GB, Granger DN, Hamilton SR, McCord JM 1982 Ischemic injury in the cat small intestine: role of superoxide radicals. Gastroenterology 82:9-15

2. Robinson JWL, Mirkovitch V 1972 The recovery of function and microcirculation in small intestinal loops following ischemia. Gut 13:784-789

3. Granger DN, Sennett M, McElearney P, Taylor AE 1980 Effect of local arterial hypotension on cat intestinal capillary permeability. Gastroenterology 79:474-480.

4. Smith EE, Crowell JW, Moran CJ, Smith RA 1967 Intestinal fluid loss in dogs during irreversible hemorrhagic shock. Surg Gynecol Obstet 125:45-48

5. Cook BH, Wilson ER, Taylor AE 1971 Intestinal fluid loss in hemorrhagic shock. Am J Physiol 221:1494-1498

6. Johansen K 1964 Regional distribution of circulating blood during submersion asphyxia in the duck. Acta Physiol Scand 62:1-9

7. Touloukian RJ, Posch JN, Spencer R 1972 The pathogenesis of ischemic gastroenterocolitis of the neonate: selective gut mucosal ischemia in asphyxiated neonatal piglets. J Pediatr Surg 7:194-205

8. Granger DN, Rutile G, McCord JM 1981 Superoxide radicals in feline intestinal ischemia. Gastroenterology 81:22-29

9. Miller DL, Schedl HP 1970 Total recovery studies of nonabsorbable indicators in the rat small intestine. Gastroenterology 58:40-46

10. Davis JS, Gander JE 1967 A reevaluation of the Roe procedure for the determination of fructose. Anal Biochem 19:72-79

11. Dalqvist A 1970 Assay of intestinal disaccharidases. Enz Biol Clin 11:52-66

12. Kramer HJ, Baker A, Kruck F 1974 Inhibition of intestinal $\left(\mathrm{Na}^{+}, \mathrm{K}^{+}\right)$ATPase in experimental uremia. Clin Chim Acta 50:13-18

13. Lowry OH, Passonneau JV A flexible system of enzymatic analysis. Academic Press, New York, 1972

14. Bradford MM 1976 A rapid and sensitive method for the quantitation of microgram quantities of protein utilizing the principle of protein dye binding. Anal Biochem 72:248-250

15. Parks DA, Bulkley GB, Granger DN 1983 Role of oxygen-derived free radicals in digestive tract disease. Surgery 94:415-422

16. Perry MA, Shepherd AP, Kvietys PR, Granger DN 1985 Effect of hypoxia on feline intestinal capillary permeability. Am J Physiol 248:G272-G276

17. Sweadner KJ, Goldin SM 1980 Active transport of sodium and potassium ions: mechanism, function and regulation. N Engl J Med 302:777-783

18. Lifshitz F, Wapnir RA, Teichberg S 1986 Alterations in jejunal transport and $\left(\mathrm{Na}^{+}, \mathrm{K}^{+}\right)$-ATPase in an experimental model of hypoxia in rats. Proc Soc Exp Biol Med 181:87-97 